\title{
Nonlinear electromagnetic modes in astrophysical plasmas with dust distributions
}

\author{
F. Verheest ${ }^{1,2}$ and T. Cattaert ${ }^{1}$ \\ 1 Sterrenkundig Observatorium, Universiteit Gent, Krijgslaan 281, 9000 Gent, Belgium \\ 2 School of Physics (Howard College Campus), University of KwaZulu-Natal, Durban 4041, South Africa
}

Received 22 January 2004 / Accepted 18 March 2004

\begin{abstract}
A derivative nonlinear Schrödinger equation is obtained for parallel electromagnetic modes in plasmas containing polydisperse charged dust. The coefficient of the dispersive term in that equation is dominated by the dust rather than the (plasma) ions, and polydisperse dust yields a larger coefficient in absolute value than an equivalent monodisperse description. This leads to a significant broadening of the nonlinear structure due to the presence of polydisperse rather than monodisperse dust, the latter contributing in itself already to a substantial increase in the width of envelope solitons compared to dust-free plasmas. When modelling the charged dust by a power-law distribution occurring in planetary ring and other astrophysical systems, it depends very much on the power-law index whether the smaller or the larger grains are more important. For certain indices the more numerous smaller grains determine the charge and mass densities, but the larger dust dominates the linear and nonlinear dispersive effects.
\end{abstract}

Key words. plasmas - waves - planets: rings - ISM: clouds

\section{Introduction}

Dust grains embedded in a plasma and radiative environment become electrically charged and their interactions with the plasma itself give rise to what is now commonly called dusty or complex plasmas. For general discussions of dusty plasmas and their many interesting properties we refer to recent monographs (Bliokh et al. 1995; Verheest 2000; Shukla \& Mamun 2002) since there now exists a vast body of original research papers that cannot possibly be cited here, with the exception of the immediately relevant ones.

In astrophysical plasmas dust distributions (in size, mass and charge) are the rule, rather than the exception, contrary to laboratory experiments that use more or less well calibrated dust grains. Hence it is of interest to incorporate polydisperse dust into the description of various modes that can exist in complex plasmas, with a particular emphasis on astrophysical applications. Some of the insight we gained in earlier studies of typical linear electrostatic (Meuris et al. 1996; Verheest et al. 2000; Jacobs et al. 2000; Yaroshenko et al. 2001; Verheest et al. 2002, 2003) and linear electromagnetic (Verheest \& Cattaert 2003) normal modes in plasmas containing dust distributions will be used in this paper to deal with nonlinear electromagnetic modes. As in ordinary plasmas, there is quite a difference in treatment between linear and nonlinear modes.

A survey of the existing literature shows that the derivative nonlinear Schrödinger (DNLS) equation is the appropriate

Send offprint requests to: $\mathrm{F}$. Verheest

e-mail: Frank.Verheest@uGent.be paradigm for parallel propagating nonlinear electromagnetic modes. The original derivations were for a simple electronion plasma, in a kinetic (Rogister 1971) or fluid (Mio et al. 1976) description, and later generalized to include several ion species and equilibrium streaming between different ion species (Verheest 1990), the latter extension having applications to the pickup of cometary ions by the solar wind in mind.

As is the case with many nonlinear evolution equations that model different physical situations, the various forms of the DNLS equations exhibit a similar mathematical structure (degree of nonlinearity, order of dispersive derivative) but with model-dependent coefficients for the terms occurring in it. In many treatments of complex plasmas all charged dust grains together are simply described as a monodisperse, additional heavy ion species with average parameters. Thus a DNLS equation with monodisperse dust has recently been shown to yield a possible mechanism for the generation of large scale structures in astrophysical plasmas containing a fraction of charged dust, as applied to large molecular clouds (Shukla \& Verheest 2003).

As explained at the beginning of this section, astrophysical complex plasmas contain polydisperse dust and we therefore aim in the present paper at incorporating a dust distribution, rather than working with average monodisperse dust. We are motivated in this by complex plasmas observed i.a. in planetary rings. Even though several papers in the literature, describing waves in dusty plasmas with polydisperse dust, start from a multi-ion description and afterwards merely replace the sum over different dust species by an integral over a continuous dust spectrum, this procedure violates the underlying assumptions 
of when a collection of ions or dusts can be treated as belonging to a number of different fluids in the first place. Hence in Sect. 2 the derivation is sketched of the appropriate form of the DNLS equation for dust distributions, with the qualifications needed to arrive at the correct form.

Section 3 then addresses some properties of the coefficient of the dispersive term in the extended DNLS equation, both for general distributions of dust charges and masses, and for specific power-law dust density distributions as encountered in e.g. planetary rings (Gurnett et al. 1983; Showalter et al. 1992; Showalter \& Cuzzi 1993), to cite but one well documented example. Such power-law distributions are fairly generic for other astrophysical plasmas as in molecular clouds, where smaller grains are more numerous than larger ones, but detailed observations outside the solar system are lacking (see Raadu 2001, and references therein). In those cases, not only densities but also charges and masses can be expressed in terms of the dust size as the defining parameter of the distribution. Finally, Sect. 4 contains our conclusions.

\section{Nonlinear formalism}

In what follows, we very briefly sketch some of the steps that lead to a DNLS equation for complex plasmas with polydisperse dust, with the emphasis on how this has to be done in a methodologically correct way. We distinguish between the electrons and ions on the one hand, treated by standard theory, and the dust distributions, described by appropriate integrals over the charge and mass distributions. In order to avoid all ambiguities, we start for the dust from a microscopic distribution function $f_{\mathrm{d}}(\boldsymbol{x}, \boldsymbol{w}, t, q, m)$ in phase space, which incorporates the dust charge $q$ and the mass $m$ as continuous, independent microscopic phase space variables, besides $\boldsymbol{x}, t$ and the phase space velocity $\boldsymbol{w}$. This is governed by an extended Vlasovtype kinetic equation (Varma 2000; Verheest et al. 2002, 2003; Verheest \& Cattaert 2003)

$\frac{\partial f_{\mathrm{d}}}{\partial t}+\boldsymbol{w} \cdot \frac{\partial f_{\mathrm{d}}}{\partial \boldsymbol{x}}+\frac{q}{m}(\boldsymbol{E}+\boldsymbol{w} \times \boldsymbol{B}) \cdot \frac{\partial f_{\mathrm{d}}}{\partial \boldsymbol{w}}=0$

where the microscopic laws of motion $\boldsymbol{w}=\dot{\boldsymbol{x}}$ and $\dot{\boldsymbol{w}}=$ $(q / m)(\boldsymbol{E}+\boldsymbol{w} \times \boldsymbol{B})$ have been used. Here $\boldsymbol{E}$ is the electric field and $\boldsymbol{B}$ the magnetic induction, with equilibrium value $\boldsymbol{B}_{0}=$ $\boldsymbol{B}_{0} \boldsymbol{e}_{z}$. For dust grains with constant charges and masses there are no $\partial\left(\dot{q} f_{\mathrm{d}}\right) / \partial q$ and $\partial\left(\dot{m} f_{\mathrm{d}}\right) / \partial m$ contributions, even though $q$ and $m$ cover a whole range.

If we were to integrate (1) over all $q, m$ and $\boldsymbol{w}$, after multiplication with some power of the microscopic velocities, we obtain the traditional chain of moment equations in physical space. As explained elsewhere (Verheest \& Cattaert 2003) the different charge and mass weightings compel one to introduce a whole sequence of velocities and higher order moments, where the same order in $w$ is combined with different powers of $q / m$, due to the magnetic part of the Lorentz force. To avoid these complications, we postpone the integration over these new phase space variables and restrict ourselves at first to integrations solely over the microscopic velocity $\boldsymbol{w}$. More details about this half-way house procedure are given elsewhere
(Verheest \& Cattaert 2004), and we define the first two velocity moments of $f_{\mathrm{d}}$ as

$g_{\mathrm{d}}=\int f_{\mathrm{d}} \mathrm{d}^{3} w$

$\boldsymbol{u}_{\mathrm{d}}=\frac{1}{g_{\mathrm{d}}} \int f_{\mathrm{d}} \boldsymbol{w} \mathrm{d}^{3} \boldsymbol{w}$.

Note that these still contain variable $q$ and $m$ values. In this sense, after a further integration over all $q$ and $m, g_{\mathrm{d}}$ will give the dust number density $n_{\mathrm{d}}$ and, likewise, $g_{\mathrm{d}} \boldsymbol{u}_{\mathrm{d}}$ will give $n_{\mathrm{d}} \boldsymbol{v}_{\mathrm{d}}$, where $\boldsymbol{v}_{\mathrm{d}}$ is the dust velocity as traditionally used in a fluid description. Hence, the first moment equations for the dust are similar to the continuity and momentum equations for ordinary plasma species, but with a different interpretation because the integrations over $q$ and $m$ still have to be done. Thus we shall be able to "translate" results obtained for electrons and ions to the corresponding results for the dust distribution. Caution is needed here, however, and it is necessary to check existing results on their applicability, before additional integrations over charge and/or mass are carried out. These occur i.e. in the expressions for the overall charge and current densities in Maxwell's equations.

In equilibrium charge neutrality demands that

$\sum_{\alpha=e, i} n_{\alpha 0} q_{\alpha}+\int g_{d 0} q \mathrm{~d} q \mathrm{~d} m=0$

and for negatively charged dust there are fewer free electrons than positive ions. Here the plasma species subscript $\alpha$ refers to the electrons $(\alpha=e)$ and the ions $(\alpha=i)$, with number densities $n_{\alpha}$, masses $m_{\alpha}$ and charges $q_{\alpha}$, where $q_{e}=-e$ and $q_{i}=e$. There is no intrinsic difficulty in treating positive dust, or even a mixture of both. In the case of positive dust there are more free electrons than ions. The usual emphasis in theoretical treatments of complex plasmas on negative dust comes from taking the standard orbital-motion-limited spherical probe theory as a model for the dust charging (Bliokh et al. 1995; Verheest 2000; Shukla \& Mamun 2002).

To derive the appropriate form of the DNLS equation, one uses the stretching of coordinates and time for electromagnetic parallel modes (Kakutani et al. 1968; Kakutani \& Ono 1969) together with a series expansion of the dependent variables in a small parameter $\varepsilon$ that measures the weakness of the dispersion and of the nonlinear effects. This method is called the reductive perturbation approach and leads to solving the set of equations to different orders in $\varepsilon$. The details of this procedure can be found in many research papers and monographs, and have been recently summarized elsewhere (Verheest 2000). One first determines the linear wave velocity $V$ in the long wavelength limit through

$V^{2}=\frac{c^{2} V_{\mathrm{A}}^{2}}{c^{2}+V_{\mathrm{A}}^{2}}$

Here a global Alfvén velocity $V_{\mathrm{A}}$ has been defined through $V_{\mathrm{A}}^{2}=B_{0}^{2} / \mu_{0} \rho_{0}$, with $\rho_{0}$ referring to the total mass density of the full plasma, electrons, ions, and dust distributions taken together,

$\rho_{0}=\sum_{\alpha=e, i} n_{\alpha 0} m_{\alpha}+\int g_{d 0} m \mathrm{~d} q \mathrm{~d} m$. 
Since for many astrophysical plasmas $V_{\mathrm{A}} \ll c$, we see that the linear phase velocity $V$ is close to $V_{\mathrm{A}}$, which corresponds either to the dust or the ion Alfvén speed, depending on how much mass there is in the dust distributions compared to the ions (and electrons).

Going to higher orders ultimately leads to the desired nonlinear evolution equation including dust distributions,

$\frac{\partial \boldsymbol{B}_{\perp}}{\partial \tau}+C \frac{\partial}{\partial \xi}\left(B_{\perp}^{2} \boldsymbol{B}_{\perp}\right)+D \boldsymbol{e}_{z} \times \frac{\partial^{2} \boldsymbol{B}_{\perp}}{\partial \xi^{2}}=\mathbf{0}$,

a DNLS equation with coefficients

$C=\frac{V^{3}}{4 B_{0}^{2} V_{A}^{2}} \simeq \frac{V_{A}}{4 B_{0}^{2}}$,

$D=\frac{\mu_{0} V^{4}}{2 B_{0}^{3}}\left(\sum_{\alpha=e, i} \frac{n_{\alpha 0} m_{\alpha}^{2}}{q_{\alpha}}+\int g_{\mathrm{d} 0} \frac{m^{2}}{q} \mathrm{~d} q \mathrm{~d} m\right)$.

Here $\boldsymbol{B}_{\perp}$ refers to the perpendicular wave magnetic induction in lowest nonzero order, orthogonal to the constant parallel equilibrium field.

Before going on, we remark that the coefficient $D$ also occurs in the small wavenumber $(k)$, small frequency $(\omega)$ expansion of the linear phase velocity

$\frac{\omega}{k}=V \pm D k+\ldots$,

which follows from the complete linear dispersion law, given elsewhere (Verheest \& Cattaert 2003). Changes in the sign of $D$ entail changes in the handedness of the circular polarization of the linear and nonlinear waves.

By projecting (6) onto two orthogonal transverse directions (the $x$ and $y$ axes) and combining the components $B_{x}$ and $B_{y}$ of $\boldsymbol{B}_{\perp}$ into a single complex variable $\varphi=B_{x}+i B_{y}$ leads to the complex scalar representation of the DNLS equation,

$\frac{\partial \varphi}{\partial \tau}+C \frac{\partial}{\partial \xi}\left(|\varphi|^{2} \varphi\right)+i D \frac{\partial^{2} \varphi}{\partial \xi^{2}}=0$.

The typical solutions of (6) or (9) are envelope solitons of the form (Mjølhus \& Wyller 1986; Mann 1988; Hada et al. 1989; Verheest \& Buti 1992; Verheest 2000)

$$
\begin{aligned}
\left|\boldsymbol{B}_{\perp}\right|^{2} & =|\varphi|^{2} \\
& =\frac{2 \gamma^{2}}{C}\left(\sqrt{\gamma^{2}+M^{2}} \cosh \frac{\gamma}{D}(\xi-M \tau)-M\right)^{-1},
\end{aligned}
$$

where $M(>0)$ represents the velocity in the co-moving frame and $\gamma$ is a parameter related to the width or to the nonlinear phase shift, depending on how one wants to characterize the soliton properties (Mjølhus \& Wyller 1986; Mann 1988; Hada et al. 1989; Verheest \& Buti 1992). All the preceding results are fully general as far as the dust distribution is concerned, and can be applied to different problems.

If one defines the width $L$ of the soliton as the width at half maximum, $|\varphi|_{\xi-M \tau=L / 2}=|\varphi|_{\max } / 2$, then one can prove that the relation between the maximum amplitude and the width obeys (Mann 1988)

$|\varphi|_{\max }^{2} L \leq \frac{8 \sqrt{3}|D|}{C}$.
Since $|\varphi|_{\max }$ is evaluated at $\xi-M \tau=0$, it is independent of $D$, so that for the width we have that $L \propto|D|$. Hence it is of importance to determine how $D$ is changed for a dust distribution compared to monodisperse dusty plasmas or to ordinary dustfree plasmas. This issue will be addressed in the next section.

\section{Discussion}

As can be seen from (7), the coefficient $C$ of the cubic nonlinear term is always positive and essentially determined by the total plasma density and the strength of the static magnetic induction. The coefficient $D$ of the dispersive term, on the other hand, requires a more delicate evaluation, since it depends on how the different ratios $\mathrm{m}^{2} / q$ are weighted.

\subsection{General remarks}

In line with previous work (Verheest et al. 2003; Verheest \& Cattaert 2003, 2004) we write averages over the equilibrium dust distribution as

$\langle\mathcal{A}\rangle=\int g_{\mathrm{d} 0} \mathcal{A} \mathrm{d} q \mathrm{~d} m$,

where $\mathcal{A}$ is a function of $q$ and $m$. We have encountered already the equilibrium dust charge density $\sigma_{\mathrm{d} 0}=\langle q\rangle$ in (3) and the dust mass density $\rho_{\mathrm{d} 0}=\langle m\rangle$ in (5). In addition, $\left\langle q^{2} / m\right\rangle / \varepsilon_{0}=$ $\omega_{p \mathrm{~d}}^{2}$ defines the correct dust plasma frequency squared, evaluated over the dust distribution, a generalization of the electron and ion plasma frequencies given through $\omega_{p \alpha}^{2}=n_{\alpha 0} q_{\alpha}^{2} / \varepsilon_{0} m_{\alpha}$. The normalization of $g_{d 0}$ itself leads to the dust number density $n_{d 0}=\langle 1\rangle$, but that is not a useful parameter to characterize polydisperse dust. And here, of course, the key quantity is $\left\langle\mathrm{m}^{2} / q\right\rangle$ occurring in $D$ in (7), and this has to be compared to the ion and electron contributions inside the same bracket.

In a previous paper (Verheest et al. 2003) we proved the general result for all possible dust distributions that $\omega_{p \mathrm{~d}}^{2} \geq$ $\sigma_{\mathrm{d} 0}^{2} / \varepsilon_{0} \rho_{\mathrm{d}}$, or equivalently, $\left\langle q^{2} / m\right\rangle\langle m\rangle \geq\langle q\rangle^{2}$, and the equality sign only obtains when all dust grains have the same charge-tomass ratio. By interchanging in the proof of that inequality the roles of $q$ and $m$ there is a similar result, namely $\left\langle m^{2} / q\right\rangle\langle q\rangle \geq$ $\langle m\rangle^{2}$. To see this explicitly, denote $g_{\mathrm{d} 0}=g_{\mathrm{d} 0}(q, m)$ and $g_{\mathrm{d} 0}^{\prime}=$ $g_{\mathrm{d} 0}\left(q^{\prime}, m^{\prime}\right)$ for brevity of notation, so that for obvious reasons of symmetry in the integration variables we have

$$
\begin{aligned}
\left\langle\frac{m^{2}}{q}\right\rangle\langle q\rangle-\langle m\rangle^{2} & =\int g_{\mathrm{d} 0} g_{\mathrm{d} 0}^{\prime}\left(\frac{m^{2} q^{\prime}}{q}-m m^{\prime}\right) \mathrm{d} q \mathrm{~d} q^{\prime} \mathrm{d} m \mathrm{~d} m^{\prime} \\
& =\int g_{\mathrm{d} 0} g_{\mathrm{d} 0}^{\prime}\left(\frac{m^{\prime 2} q}{q^{\prime}}-m m^{\prime}\right) \mathrm{d} q \mathrm{~d} q^{\prime} \mathrm{d} m \mathrm{~d} m^{\prime}
\end{aligned}
$$

This immediately leads to

$$
\begin{aligned}
\left\langle\frac{m^{2}}{q}\right\rangle\langle q\rangle & -\langle m\rangle^{2}= \\
& \frac{1}{2} \int g_{\mathrm{d} 0} g_{\mathrm{d} 0}^{\prime} q q^{\prime}\left(\frac{m}{q}-\frac{m^{\prime}}{q^{\prime}}\right)^{2} \mathrm{~d} q \mathrm{~d} q^{\prime} \mathrm{d} m \mathrm{~d} m^{\prime},
\end{aligned}
$$

which is always non-negative. 
Interestingly, both inequalities are special cases of an even more general property of the dust distributions. Writing moments as $\varpi_{\mathrm{d}}^{(\ell)}=\left\langle q^{\ell+1} / m^{\ell}\right\rangle$, one can prove for all $\ell$ that

$$
\begin{aligned}
& \varpi_{\mathrm{d}}^{(\ell+2)} \varpi_{\mathrm{d}}^{(\ell)}-\left(\varpi_{\mathrm{d}}^{(\ell+1)}\right)^{2}= \\
& \frac{1}{2} \int g_{\mathrm{d} 0} g_{\mathrm{d} 0}^{\prime} \frac{q^{\ell+1} q^{\prime \ell+1}}{m^{\ell} m^{\prime \ell}}\left(\frac{q}{m}-\frac{q^{\prime}}{m^{\prime}}\right)^{2} \mathrm{~d} q \mathrm{~d} q^{\prime} \mathrm{d} m \mathrm{~d} m^{\prime} .
\end{aligned}
$$

These are all non-negative, and include as special cases $\left\langle q^{2} / m\right\rangle\langle m\rangle \geq\langle q\rangle^{2}$ for $\ell=-1$ and $\left\langle m^{2} / q\right\rangle\langle q\rangle \geq\langle m\rangle^{2}$ for $\ell=-2$

Now we are ready to discuss $D$. Because of the heavy mass weighting, the electron contribution can be neglected compared to the ion term. In standard plasmas without charged dust, $D$ is determined from the (positive) ion properties and $D$ is positive.

To see how $D$ is influenced by a dust distribution, we first look at the simplified case that there are no free electrons, which implies that the dust is negatively charged. Since equilibrium charge neutrality (3) then indicates that $|\langle q\rangle|=n_{i 0} e$, it follows from (14) that in (7), the definition of $D$, the ratio of the two terms between brackets obeys

$\frac{\left|\left\langle m^{2} / q\right\rangle\right|}{n_{i 0} m_{i}^{2} / e} \geq \frac{\langle m\rangle^{2}}{n_{i 0}^{2} m_{i}^{2}}=\left(\frac{\rho_{\mathrm{d} 0}}{\rho_{i 0}}\right)^{2}$.

Here $\rho_{i 0}=n_{i 0} m_{i}$ is the equilibrium ion mass density. Introducing an average dust charge $\bar{q}_{\mathrm{d}}=\langle q\rangle / n_{\mathrm{d} 0}$, with an absolute charge number $\bar{Z}_{\mathrm{d}}=\left|\bar{q}_{\mathrm{d}}\right| / e$, and an average dust mass $\bar{m}_{\mathrm{d}}=\langle m\rangle m / n_{\mathrm{d} 0}$, we have that

$$
\frac{\rho_{\mathrm{d} 0}}{\rho_{i 0}}=\frac{\bar{m}_{\mathrm{d}}}{\bar{Z}_{\mathrm{d}} m_{i}}
$$

For all known dusty plasmas the mass-per-charge of charged dust grains largely exceeds the ion mass, which will also hold for the averages used here. Therefore $\rho_{\mathrm{d} 0} / \rho_{i 0}$ is usually a large ratio indeed, and $D$ is dominated by the dust contribution. Thus $D$ is larger than it would be without charged dust, and negative, moreover. This means that the width of the envelope solitons is much larger than in standard plasmas.

The inclusion of free electrons changes the previous comparison into

$$
\frac{\left|\left\langle m^{2} / q\right\rangle\right|}{n_{i 0} m_{i}^{2} / e} \geq\left(1-\frac{n_{e 0}}{n_{i 0}}\right)\left(\frac{\bar{m}_{\mathrm{d}}}{\bar{Z}_{\mathrm{d}} m_{i}}\right)^{2},
$$

using $|\langle q\rangle|=\left(n_{i 0}-n_{e 0}\right) e$. Hence it is only at extremely low dust densities that this ratio can be smaller than 1 . For all interesting cases where charged dust plays indeed a non-negligible role, the coefficient $D$ of the dispersive term is dominated by the dust. Moreover, since $\left|\left\langle m^{2} / q\right\rangle\right| \geq\langle m\rangle^{2} / \mid\langle q\rangle$, it follows that

$\left|\left\langle\frac{m^{2}}{q}\right\rangle\right| \geq \frac{n_{\mathrm{d} 0} \bar{m}_{\mathrm{d}}^{2}}{\left|\bar{q}_{\mathrm{d}}\right|}$.

Hence a dust distribution yields a larger $\left|\left\langle m^{2} / q\right\rangle\right|$ value, thus further increasing the (absolute) value of $D$ and the width of the envelope solitons, than if the corresponding quantity were computed for monodisperse dust with average charges and masses, as used e.g. by Shukla \& Verheest (2003).

\subsection{Size distributions}

Now we assume that the dust grains all have equilibrium values determined from the orbital-motion-limited or spherical probe charging theory (see e.g. Bliokh et al. 1995; Verheest 2000; Shukla \& Mamun 2002) where the dust is charged by electron and ion currents to the grains, and the charge is proportional to the grain size $a$ in a given plasma environment. Furthermore, for grains of similar composition the mass varies with $a^{3}$. Thus $q(a)=Q a$ and $m(a)=M a^{3}$, so that we are actually dealing with a dust size distribution. In the orbital-motion-limited model the dust gets negatively charged and $Q=-|Q|<0$.

The (redefined) distribution function $f_{\mathrm{d}}(\boldsymbol{x}, \boldsymbol{w}, t, a)$ will now depend on $a$ as the parameter characterizing the dust distribution, instead of on $q$ and $m$ separately. The equilibrium densities $\varpi_{d}^{(\ell)}$ are thus given by

$\varpi_{\mathrm{d}}^{(\ell)}=\frac{Q^{\ell+1}}{M^{\ell}} \int f_{\mathrm{d} 0}(\boldsymbol{w}, a) a^{1-2 \ell} \mathrm{d}^{3} \boldsymbol{w} \mathrm{d} a$.

Next we take a standard power-law distribution, i.e. we assume that

$g_{\mathrm{d} 0}(a)=\int f_{\mathrm{d} 0}(\boldsymbol{w}, a) \mathrm{d}^{3} \boldsymbol{w}=K a^{-\beta}$

in an interval $\left[a_{\min }, a_{\max }\right]$. Such power-law density decreases with size are typical for different space plasma environments. Distributions of this sort have been observed in planetary ring plasmas, with power-law indices $\beta=4.6$ for the $\mathrm{F}$ ring of Saturn (Showalter et al. 1992) and $\beta=6$ (Showalter \& Cuzzi 1993), or $\beta=7$ (Gurnett et al. 1983) for the G ring. Moreover, for typical solar system dust size distributions the parameter $a_{\min } / a_{\max }$ is usually very small. We will use that to simplify some of the subsequent expressions, and consider $\beta$ values that lie between 3 and 7. Such power-law distributions are fairly generic for other astrophysical plasmas, as in molecular clouds, based on considerations that smaller grains are more numerous than larger ones, although detailed observations outside the solar system are lacking for the time being.

Combining (20) and (21) gives

$$
\begin{aligned}
\varpi_{\mathrm{d}}^{(\ell)} & =\frac{K Q^{\ell+1}}{M^{\ell}} \int_{a_{\min }}^{a_{\max }} a^{1-\beta-2 \ell} \mathrm{d} a \\
& \propto \begin{cases}\frac{a_{\max }^{2-\beta-2 \ell}-a_{\min }^{2-\beta-2 \ell}}{2-\beta-2 \ell} & \text { if } 2-2 \ell \neq \beta, \\
\ln \frac{a_{\max }}{a_{\min }} & \text { if } 2-2 \ell=\beta .\end{cases}
\end{aligned}
$$

The generic expressions for these integrals over dust size are weighted towards the larger sizes when $2-2 \ell>\beta$, and towards the smaller sizes when $2-2 \ell<\beta$. The latter are unfortunately difficult to ascertain observationally, since they could be well below the sensitivity of the detectors.

Given that for dust distributions the correct definition of the square of the dust plasma frequency is $\omega_{p \mathrm{~d}}^{2}=\left\langle q^{2} / m\right\rangle / \varepsilon_{0}=\varpi_{\mathrm{d}}^{(1)} / \varepsilon_{0}$, the critical value would be here $\beta=0$, which does not correspond to a realistic power law. Hence the dust plasma frequency, needed e.g. for a discussion of dust-acoustic waves (Rao et al. 1990), is always weighted 
towards the more numerous but smaller dust grains. Next, we have encountered the equilibrium dust charge density, $\sigma_{\mathrm{d} 0}=$ $\langle q\rangle=\varpi_{\mathrm{d}}^{(0)}$, for which the critical value is $\beta=2$, and the dust mass density $\rho_{\mathrm{d} 0}=\langle m\rangle=\varpi_{\mathrm{d}}^{(-1)}$, with a critical value of $\beta=4$.

For this paper the important quantity is $\left\langle m^{2} / q\right\rangle=\varpi_{\mathrm{d}}^{(-2)}$, determining the behaviour of $D$, and the critical value has now reached $\beta=6$. Thus for the $\mathrm{F}$ ring of Saturn, with power-law index $\beta=4.6$ (Showalter et al. 1992), we can conclude that the dust charge and mass densities are determined chiefly by the smaller dust grains, but it is the larger dust grains (with correspondingly larger mass-per-charge ratios) that dominate the coefficient $D$ of the dispersive term in the DNLS Eq. (6).

One of the values $\beta=6$ (Showalter \& Cuzzi 1993) for the $\mathrm{G}$ ring of Saturn is the critical one for $\left\langle\mathrm{m}^{2} / q\right\rangle$, so that also the ratio $a_{\max } / a_{\min }$ of the extreme sizes (for which the powerlaw distribution holds) plays an important role. However, other values have been quoted for the G ring, like $\beta=7$ (Gurnett et al. 1983), and then all quantities of importance to us in the present discussion will be dominated by the more numerous, smaller grains at the lower end of the dust size spectrum.

The Voyager observations that have yielded these powerlaw indices and related information about planetary dust in the rings of the Jovian planets are now almost twenty years old. However exciting these observations may have been, they essentially correspond to snapshots from the flybys of the Voyager spacecraft on their Grand Tour of the solar system. Hence we look forward with much anticipation to the more extended and precise data that the Cassini mission, once in orbit around Saturn, will start to beam to us from the middle of 2004 onwards. Other methods will be needed to quantify the dust distributions outside the heliosphere.

\section{Conclusions}

For nonlinear parallel electromagnetic waves in complex plasmas with dust distributions we derived the correct form of the DNLS equation and found that in the coefficient of the dispersive term it is the dust that dominates over the plasma ion and electron contributions, because of the heavier dust massto-charge ratios. This is a logical extension of what was known for dust-free multispecies plasmas, where the heavier ions tend to dominate, provided they have a reasonable number density. Moreover, in absolute value, a polydisperse dust distribution yields a larger coefficient of the dispersive term than an equivalent monodisperse dust description would give at average charges and masses. This could lead to a significant broadening of the nonlinear structure due to the presence of polydisperse rather than monodisperse dust, the latter contributing in itself already to a substantial increase in the width of the envelope solitons compared to dust-free plasmas.

When modelling the charged dust by a power-law distribution, we found in addition that it will depend very much on the power-law index whether the smaller or the larger grains are more important. There are indices for which the more numerous smaller grains determine the charge and mass densities, and hence also the average charges and masses, but the larger dust dominates the linear and nonlinear dispersive effects. The present observational data from the Voyager missions, however valuable, are not extensive and precise enough to allow us a more definite statement or more quantitative results. That will have to wait for the coming Cassini data flowing from Saturn in the middle of 2004.

Acknowledgements. This work was supported by the Fonds voor Wetenschappelijk Onderzoek (Vlaanderen). Interesting discussions with N. F. Cramer and V. V. Yaroshenko are gratefully acknowledged.

\section{References}

Bliokh, P., Sinitsin, V., \& Yaroshenko, V. 1995, Dusty and SelfGravitational Plasmas in Space (Dordrecht: Kluwer)

Gurnett, D. A., Grün, E., Gallagher, D., Kurth, W. S., \& Scarf, F. L. 1983, Icarus, 53, 236

Hada, T., Kennel, C. F., \& Buti, B. 1989, J. Geophys. Res., 94, 65

Jacobs, G., Verheest, F., Hellberg, M. A., \& Pillay, S. R. 2000, Phys. Plasmas, 7, 4390

Kakutani, T., \& Ono, H. 1969, J. Phys. Soc. Japan, 26, 1305

Kakutani, T., Ono, H., Taniuti, T., \& Wei, C.-C. 1968, J. Phys. Soc. Japan, 24, 1159

Mann, G. 1988, J. Plasma Phys., 40, 281

Meuris, P., Verheest, F., \& Lakhina, G. S. 1996, Plan. Space Sci., 45, 449

Mio, K., Ogino, T., Minami, K., \& Takeda, S. 1976, J. Phys. Soc. Japan, 41, 265

Mjølhus, E., \& Wyller, J. 1986, Phys. Scr., 33, 442

Raadu, M. A. 2001, IEEE Trans. Plasma Sci., 29, 182

Rao, N. N., Shukla, P. K., \& Yu, M. Y. 1990, Planet. Space Sci., 38, 543

Rogister, A. 1971, Phys. Fluids, 14, 2733

Showalter, M. R., \& Cuzzi, J. N. 1993, Icarus, 103, 124

Showalter, M. R., Pollack, J. B., Ockert, M. E., Doyle, L. R., \& Dalton, J. B. 1992, Icarus, 100, 394

Shukla, P. K., \& Mamun, A. A. 2002, Introduction to Dusty Plasma Physics (Bristol: IOP)

Shukla, P. K., \& Verheest, F. 2003, A\&A, 401, 849

Varma, R. K. 2000, Phys. Plasmas, 7, 3885

Verheest, F. 1990, Icarus, 86, 273

Verheest, F. 2000, Waves in Dusty Space Plasmas (Dordrecht: Kluwer)

Verheest, F., \& Buti, B. 1992, J. Plasma Phys., 47, 15

Verheest, F., \& Cattaert, T. 2003, Phys. Plasmas, 10, 956

Verheest, F., \& Cattaert, T. 2004, Waves in complex plasmas with dust distributions (charge/size/mass) revisited, IEEE Trans. Plasma Sci., 32, in press

Verheest, F., Jacobs, G., \& Yaroshenko, V. V. 2000, Phys. Plasmas, 7, 3004

Verheest, F., Yaroshenko, V. V., \& Hellberg, M. A. 2002, Phys. Plasmas, 9, 2479

Verheest, F., Hellberg, M. A., \& Yaroshenko, V. V. 2003, Phys. Rev. E, 67, 016406

Yaroshenko, V. V., Jacobs, G., \& Verheest, F. 2001, Phys. Rev. E, 64, 036401 